\title{
Synchronization of Neuronal Networks via Control Rank Pinning Scheme
}

\author{
Qingying Miao, ${ }^{1}$ W. K. Wong, ${ }^{2}$ and Dan Shan ${ }^{3}$ \\ ${ }^{1}$ School of Continuing Education, Shanghai Jiao Tong University, Shanghai 200030, China \\ ${ }^{2}$ Institute of Textiles and Clothing, The Hong Kong Polytechnic University, Hong Kong \\ ${ }^{3}$ College of Information Science and Technology, Dong Hua University, Shanghai 201620, China
}

Correspondence should be addressed to Qingying Miao; qymiao@situ.edu.cn

Received 11 January 2013; Accepted 26 January 2013

Academic Editor: Yang Tang

Copyright (C) 2013 Qingying Miao et al. This is an open access article distributed under the Creative Commons Attribution License, which permits unrestricted use, distribution, and reproduction in any medium, provided the original work is properly cited.

\begin{abstract}
Recent studies have proposed the controlling regions in the corticocortical network of cats' brain at different scales. Here we study pinning control using a simple model of coupled oscillators assigned to cortical areas in the corticocortical network of cats' brain. We analyze control rank (CR) values of areas in the cortical network. It is found that most of the hubs have bigger CR values than other nodes in the same functional community. Moreover, we analyze the synchrony state of the functional communities in the cortical network, revealing that a community with a larger percentage of pinned nodes inside will be conducive to synchronization.
\end{abstract}

\section{Introduction}

Over the last years, the complex networks approach has been widely applied in the field of neuroscience. The study changes from the topological structure of the global neuronal networks to the segregation and the integration of different subnetworks. The analysis of the anatomical connectivity of the mammalian cortex has uncovered that large-scale neuronal networks display high clustering and short path length $[1,2]$. The cortical network also shows a hierarchy of complex connectivity and a hierarchy of interacting elements on different levels: the microscopic level of interacting neurons, the mesoscopic level of local neural circuits, and the macroscopic level of large-scale organization of the brain areas [3-6]. The understanding of the topological structure in different levels of neural networks helpf in studying the dynamical process on such networks.

Synchronization is widely observed in many fields such as coupled nonlinear systems and neural networks, which closely relates to neural information processing [7-12]. It is found that the synchronization phenomena, over a wide range of spatial and temporal scales, reflect the relevance for cognitive dysfunctions and pathophysiology [13]. The process of synchronization is from partial nodes to the global network, and it also reflects the underlying topology of interactions [14]. Pinning control is an effective method to attain synchronization of networks [15-25]. By injecting feedback control action on part of the nodes, the network is synchronized to equilibrium state or nonlinear dynamical orbits. Utilizing control theory and master stability function (MSF) method, the concept of controllability was proposed to evaluate how easily a network is controllable [21]. The Eigenratio $R$ and the maximum imaginary part $\sigma$ of an extended matrix were proposed to evaluate the network's controllability. Both the number of pinned nodes and control gains influence the network's synchronization. The detection of controlling regions was converted into a constrained optimization problem, where the objective function $R$ was minimized and $\sigma$ was regarded as a constraint [22-24]. By utilizing control theory and assuming linear dynamics in each vertex, the results of structure controllability \pm revealed that, in both model and real systems, the driver nodes tend to avoid the high-degree nodes $[25,26]$. The concept of control rank (CR) was defined and used to control a scalefree (SF) directed dynamical network to an equilibrium state [27]. 
In this work, we study synchronization in the cortical network of cats' brain. Each cortical area is modeled by a nonlinear dynamical system with different initial states. The coupling between areas is modeled using the inner state coupling of the systems' state variables. Based on the network model, we analyze CR values of each area in the cortical network. Then we study CR pinning scheme in terms of the number and the distributions of the pinned nodes. Moreover, we analyze global synchronization and community synchronization in the cortical network.

The remainder of this paper is organized as follows. In Section 2, the topic of pinning control is introduced. Then, we introduce CR pinning scheme in Section 3. In Section 4, we present CR values of each area in the cortical network. In Section 5, we analyze synchronization under CR pinning scheme. Conclusions are given in Section 6.

\section{Overview of Pinning Control}

Notations. Throughout this paper, define a graph by $\mathscr{G}=$ $[\mathscr{V}, \mathscr{E}]$, where $\mathscr{V}=\{1, \ldots, N\}$ denotes the vertex set and $\mathscr{E}=\{e(i, j)\}$ denotes the edge set.

We consider a general complex network model composed of $N$ identical nodes with diffusively coupling. The model is described as follows:

$$
\dot{x}_{i}=f\left(x_{i}\right)-c \sum_{j=1}^{N} g_{i j} \Gamma x_{j}, \quad i \in \mathscr{V},
$$

where $x_{i}=\left(x_{i 1}, x_{i 2}, \ldots, x_{i n}\right)^{T}$ is the state variable of the $i$ th node; $f\left(x_{i}\right)$ is a continuous vector function; the positive constant $c>0$ is the overall coupling strength; $\Gamma=$ $\operatorname{diag}\left(r_{1}, r_{2}, \ldots, r_{n}\right)$ is the inner coupling matrix and reveals the coupling terms of two connected nodes. Connection matrix $G=\left(g_{i j}\right) \in \mathbb{R}^{N \times N}$ is related to the topology of the network and represents the coupling configuration of the network. If there is a directed link from node $j$ to $i(i \neq j)$ and the weight of the link is $w_{i j}$, then $g_{i j}=w_{i j}$, otherwise $g_{i j}=0$. Let $\mathrm{OD}(i)$ and $\operatorname{ID}(i)$ denote the output degree (outdegree) and input degree (indegree) of the $i$ th node, respectively. The outdegree $\mathrm{OD}(i)=\sum_{j=1, j \neq i}^{N} g_{j i}, i \in \mathscr{V}$ of a node $i$ is the number of efferent connections that it projects to other nodes, and its indegree $\operatorname{ID}(i)=\sum_{j=1, j \neq i}^{N} g_{i j}, i \in \mathscr{V}$, is the number of the afferent connections it receives. In this paper, the matrix $G$ is asymmetric, and it has complex eigenvalues. Assume, the eigenvalues set of matrix $G$ is $\mu_{i}=\mu_{i}^{r}+j \mu_{i}^{m}(j=\sqrt{-1}), i \in \mathscr{V}$, and they are ordered in such a way that $\mu_{1}^{r} \leq \mu_{2}^{r} \leq \cdots \leq$ $\mu_{N}^{r}$. The Laplacian matrix of the directed network (1) can be defined as $L=-G, L_{i i}=\sum_{j=1, j \neq i}^{N} L_{i j}, i \in \mathscr{V}$. Thus, the matrix has $\sum_{j=1}^{N} L_{i j}=0, i \in \mathscr{V}$.

Assume, $s(t)$ is the desired target trajectory and satisfies

$$
\dot{s}(t)=f(s(t)) .
$$

Applying pinning control to (1) to control the network to desired reference evolution $s(t)$, we get the following expression:

$$
\begin{gathered}
\dot{x}_{i}=f\left(x_{i}\right)-c \sum_{j=1}^{N} g_{i j} \Gamma\left(x_{j}\right)+u_{i}, \quad i=1,2, \ldots, l, \\
\dot{x}_{i}=f\left(x_{i}\right)-c \sum_{j=1}^{N} g_{i j} \Gamma\left(x_{j}\right), \quad i=l+1, l+2, \ldots, N,
\end{gathered}
$$

where $u_{i}=-k_{i} \Gamma\left(x_{i}-s\right), k_{i}>0, i=1,2, \ldots, l . k_{i}$ are control gains and assumed to be constant here, for example, $k_{i}=k, i=1,2, \ldots, l$. Let $p$ denote the ratio of the pinned nodes over all nodes of the network $(0 \leq p \leq 1)$, then the pinned nodes set $I=\left\{i_{1}, i_{2}, \ldots, i_{l}\right\}$, where $l=\lfloor p \times N\rfloor$. Here, assume that for any node $i(i \in[1, N])$, we can always find a node $j$ in the pinned nodes set $I$ such that there is a directed path from the pinned node $j$ to node $i \quad(i \neq j)$.

Assume that $s(t)$ is a virtual system and by setting $y_{i}(t)=$ $x_{i}(t), i \in \mathscr{V}$, and $y_{N+1}(t)=s(t)$, we obtain a network with $N+1$ dynamical systems as follows [21]:

$$
\dot{y}_{i}=f\left(y_{i}\right)-c \sum_{j=1}^{N+1} M_{i j} \Gamma\left(y_{j}\right), \quad i=1,2, \ldots, N+1
$$

where $M=\left\{M_{i j}\right\}$ is an $(N+1)$-dimensional squared matrix defined by

$$
M=\left(\begin{array}{ccccc}
L_{11}+\delta_{1} k_{1} & L_{12} & \ldots & L_{1 N} & -\delta_{1} k_{1} \\
& & & & \\
L_{21} & L_{22}+\delta_{2} k_{2} & \ldots & L_{2 N} & -\delta_{2} k_{2} \\
\vdots & \vdots & \ddots & \ldots & \vdots \\
L_{N 1} & L_{N 2} & \ldots & L_{N N}+\delta_{N} k_{N} & -\delta_{N} k_{N} \\
0 & 0 & \ldots & 0 & 0
\end{array}\right),
$$

where if $i \in I, \delta_{i}=1$, else $\delta_{i}=0$. Through the above transformation from (1) to (6), the problem of controllability of complex networks is converted into synchronizability of networks. Let $\lambda_{i}^{r}+j \lambda_{i}^{m}(j=\sqrt{-1})$ be the eigenvalues of the matrix $M$ and in the order of $0=\lambda_{1}^{r} \leq \lambda_{2}^{r} \leq \cdots \leq$ $\lambda_{N+1}^{r}$. Similar to the analysis method of synchronizability of the networks, the bounded systems' controllability can be evaluated by eigenvalues ratio

$$
\begin{gathered}
R=\frac{\lambda_{N+1}^{r}}{\lambda_{2}^{r}}, \\
\sigma=\max \left\{\lambda_{i}^{m}\right\}, \quad i=1,2, \ldots, N+1 .
\end{gathered}
$$

The smaller the $R$ and $\sigma$ are, the easier the network is controllable. It is evident that the values of control gains $k$ and the number of pinned nodes $l$ will influence the eigenvalues of matrix $M$. 


\section{CR Pinning Scheme}

Usually, two kinds of pinning control strategies are considered on general network model. (i) Random pinning: the pinned nodes are randomly selected with uniform probability. (ii) Selective pinning: the nodes are sorted according to a certain property of the nodes, for instance, the nodes' degree, betweenness, or closeness centrality, then the pinned nodes are chosen according to the order. It is mentioned that random pinning scheme cannot guarantee synchronization of the directed networks [20]. It is verified that synchronization can be achieved if and only if forcing is applied to the roots of trees in a spanning-directed forest of the interaction graph of connection matrix $G$.

Supposing that each node with indegree has contribution to the nodes point to it, so the nodes which have more children and grandchildren and such as this will have larger $\mathrm{CR}$ values than others. According to $\mathrm{CR}$ values of each node, the concept of CR pinning scheme was defined and utilized to deal with pinning control of the directed networks [27].

Supposing that $\left[\mathrm{CR}^{0}\right]_{N \times 1}$ is a row vector composed of initial CR values of each node. After $k$ times iteration, the final stable $C R$ values $\mathrm{CR}^{k}$ are calculated as follows:

$$
\mathrm{CR}^{k}=\mathrm{CR}^{k-1} \times \mathrm{ID}^{-1} \times L,
$$

where $\mathrm{ID}=\operatorname{diag}\left(\mathrm{ID}_{1}, \mathrm{ID}_{2}, \ldots, \mathrm{ID}_{N}\right)$. At each iteration in (7), vertex $j$ will contribute with $\mathrm{CR}_{j} \times\left(1 / \mathrm{ID}_{j}\right)$ to each of its indegree neighbors, which is in direct proportion to $1 / \mathrm{ID}_{j}$. If there is a path from vertex $p$ to $j$ through vertex $i, j$ will contribute with $\mathrm{CR}_{j} \times\left(1 / \mathrm{ID}_{i}\right) \times\left(1 / \mathrm{ID}_{j}\right)$ to the $\mathrm{CR}$ value of vertex $p$. Hence, the contribution of CR value from vertex $j$ to vertex $p$ attenuates with increment of the path length from $p$ to $j$. The CR values have an obvious positive correlation with the nodes' outdegree. It is also related to the largest distance from it to all the other vertices. Considering both the property of outdegree and the distance from the pinned nodes to the others in the network, pinning a vertex according to its CR value will be an effective strategy in dealing with control of directed networks.

\section{The Cortical Network of Cats' Brain}

The cortical network of cats' brain can be divided into 53 cortical areas, linked by about 830 fibres of different densities into a weighted and directed complex network. Here we use a data set from [28]. The cerebral cortex consists of four topological interconnected communities that agree with four functional cortical subdivisions: visual cortex $(\mathrm{V}, 16$ areas), Auditory (A, 7 areas), somatomotor (SM, 16 areas), and frontolimbic (FL, 14 areas). The auditory community is sparsely connected, while the visual, somatomotor, and frontolimbic are densely connected among each other.

In the cortical network, the hub areas (Hubs) are densely interconnected at the top of the cortical hierarchy. Here we consider the case that the Hubs comprise 11 nodes from four functional communities, 20a, 7, and AES from visual cortex, $\mathrm{EPp}$ from auditory, $6 \mathrm{~m}$ and 5AI from somatosensory-motor, and Ia, Ig, CGp, 35, and 36 from frontolimbic [29].
TABLE 1: CR value of areas of the cortical network with size $N=53$.

\begin{tabular}{|c|c|c|c|}
\hline CR Order & Name & CR Value & Community \\
\hline 1 & CGp & 0.04478 & Frontolimbic \\
\hline 2 & $5 \mathrm{Al}$ & 0.03849 & Somato-Motor \\
\hline 3 & $\mathrm{CGa}$ & 0.03760 & Frontolimbic \\
\hline 4 & Ia & 0.03692 & Frontolimbic \\
\hline 5 & $6 \mathrm{~m}$ & 0.03499 & Somato-Motor \\
\hline 6 & 7 & 0.03319 & Visual \\
\hline 7 & $5 \mathrm{Bm}$ & 0.03234 & Somato-Motor \\
\hline 8 & $5 \mathrm{Am}$ & 0.03125 & Somato-Motor \\
\hline 9 & PFCMd & 0.03114 & Frontolimbic \\
\hline 10 & $5 \mathrm{Bl}$ & 0.02990 & Somato-Motor \\
\hline 11 & 35 & 0.02973 & Frontolimbic \\
\hline 12 & PFCL & 0.02952 & Frontolimbic \\
\hline 13 & $20 \mathrm{a}$ & 0.02939 & Visual \\
\hline 14 & $\operatorname{Ig}$ & 0.02919 & Frontolimbic \\
\hline 15 & SSAo & 0.02887 & Somato-Motor \\
\hline 16 & AES & 0.02766 & Visual \\
\hline 17 & $4 g$ & 0.02667 & Somato-Motor \\
\hline 18 & 61 & 0.02564 & Somato-Motor \\
\hline 19 & $\mathrm{EPp}$ & 0.02558 & Auditory \\
\hline 20 & SSSAi & 0.02450 & Somato-Motor \\
\hline 21 & 36 & 0.02136 & Frontolimbic \\
\hline 22 & SIV & 0.02131 & Somato-Motor \\
\hline 23 & PFCMil & 0.02091 & Frontolimbic \\
\hline 24 & $20 \mathrm{~b}$ & 0.01867 & Visual \\
\hline 25 & SII & 0.01847 & Somato-Motor \\
\hline 26 & 4 & 0.01825 & Somato-Motor \\
\hline 27 & 19 & 0.01732 & Visual \\
\hline 28 & RS & 0.01680 & Frontolimbic \\
\hline 29 & Enr & 0.01608 & Frontolimbic \\
\hline 30 & PLLS & 0.01329 & Visual \\
\hline 31 & PMLS & 0.01307 & Visual \\
\hline 32 & $3 b$ & 0.01297 & Somato-Motor \\
\hline 33 & PS & 0.01292 & Visual \\
\hline 34 & 18 & 0.01070 & Visual \\
\hline 35 & $\mathrm{P}$ & 0.01064 & Auditory \\
\hline 36 & $21 \mathrm{a}$ & 0.01059 & Visual \\
\hline 37 & $3 a$ & 0.01018 & Somato-Motor \\
\hline 38 & 17 & 0.00997 & Visual \\
\hline 39 & AII & 0.00952 & Auditory \\
\hline 40 & 2 & 0.00887 & Somato-Motor \\
\hline 41 & AMLS & 0.00881 & Visual \\
\hline 42 & 1 & 0.00881 & Somato-Motor \\
\hline 43 & AI & 0.00840 & Auditory \\
\hline 44 & $21 b$ & 0.00796 & Visual \\
\hline 45 & VLS & 0.00751 & Visual \\
\hline 46 & ALLS & 0.00618 & Visual \\
\hline 47 & DLS & 0.00598 & Visual \\
\hline 48 & $\mathrm{pSb}$ & 0.00594 & Frontolimbic \\
\hline 49 & $\mathrm{Sb}$ & 0.00550 & Frontolimbic \\
\hline 50 & $\mathrm{VP}(\operatorname{ctx})$ & 0.00438 & Auditory \\
\hline 51 & Tem & 0.00430 & Auditory \\
\hline 52 & AAF & 0.00381 & Auditory \\
\hline 53 & Hipp & 0.00318 & Frontolimbic \\
\hline
\end{tabular}




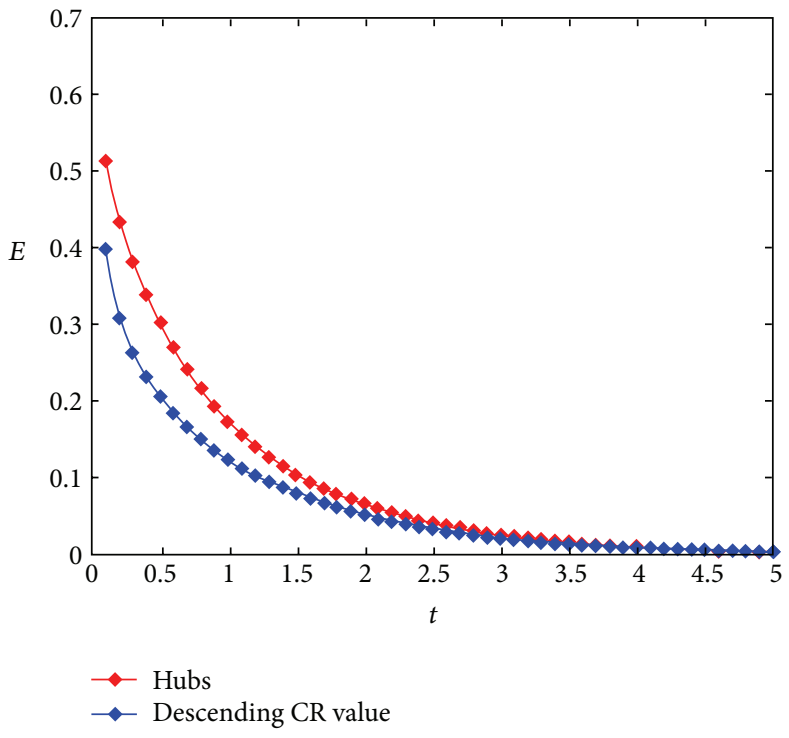

(a)

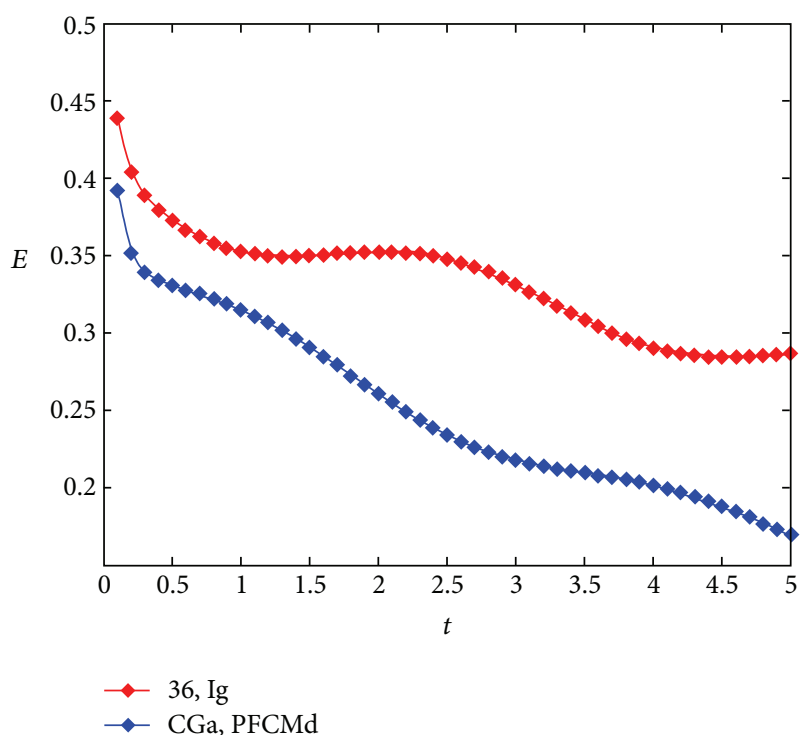

(b)

FIGURE 1: Time evolution of pinning control error $E$ for the cortical network with $N=53$. The coupling strength $c=0.3$, and the feedback gains $k=30$. (a) $p=0.2$. Red line is used in the case of pinning the Hubs, and blue line is used in the case of descending CR value pinning scheme. (b) $p=0.03$. Red line is used in the case that Ig and 36 are pinned, and blue line is used in the case that CGa and PFCMd are pinned.

We present the CR values of 53 areas of the cortical network in Table 1 . The CR values are sorted in a descending way. The area named CGp in frontolimbic has the biggest CR value. It is impressive that most areas in auditory have smaller CR values. Taking an analysis of the CR values of the Hubs, we find that the Hubs in visual, auditory, somatosensory-motor communities have bigger CR values than the others in the same community. But for frontolimbic, the hub areas are Ig and 36 other than CGa and PFCMd, which have bigger CR values than the two areas mentioned before.

From the viewpoint of a control scheme design, we compare the effect of pinning the Hubs and the nodes based on descending CR values. As shown in Figures 1(a) and 1(b), the pinning scheme according to descending the $\mathrm{CR}$ value has an advantage at the beginning of pinning control, then it becomes consistent with pinning the Hubs till all nodes synchronize to $s(t)$. Moreover, a further analysis between $\mathrm{CGa}, \mathrm{PFCMd}, \mathrm{Ig}$, and 36 in frontolimbic is presented. We study the dynamics via pinning only two nodes of CGa and PFCMd or Ig and 36. From Figure 1(b), it is evident that CGa and PFCMd have better property in controlling the network.

\section{Analysis of CR Pinning Scheme}

In this section, we analyze the descending CR pinning scheme based on the cortical network described in Section 4 . The dynamics of each area are modeled by Rössler's system. The Rössler system is given by the following differential equations:

$$
\begin{gathered}
\dot{x}_{1}=-x_{2}-x_{3}, \\
\dot{x}_{2}=x_{1}+a x_{2}, \\
\dot{x}_{3}=b+x_{3}\left(x_{1}-c\right),
\end{gathered}
$$

where $a, b$, and $c$ are constants. In this study, $a=0.165$, $b=0.2$, and $c=10$ are used. The output function $\Gamma=((1$ $\left.\left.\begin{array}{ll}0 & 0\end{array}\right),\left(\begin{array}{lll}0 & 1 & 0\end{array}\right),\left(\begin{array}{lll}0 & 0 & 1\end{array}\right)\right)$ indicates that the systems are coupled through the variables $x_{i 1}, x_{i 2}$, and $x_{i 3}$. Here we evaluate the effects of $n$ pinned nodes with feedback gains $k_{1}=k_{2}=\cdots=$ $k_{n}$ on the control error $E$. The control error is denoted by $E=(1 /(\Delta T) N) \sum_{i=1}^{N} \int_{T}^{T+\Delta T}\left\|x_{i}(t)-s(t)\right\| d t$, in which $\left\|x_{i}\right\|=$ $\left|x_{i 1}\right|+\left|x_{i 2}\right|+\left|x_{i 3}\right|$.

Next, we present numerical results of the descending CR pinning scheme under variations of the coupling strength $c$ and the pinning probability $p$ on the cortical network.

Control error $E$ has been calculated in Figures 2(a) and 2 (b) on networks with $c=0.1,0.2,0.3$, and 0.5 (pinning ratio $p=0.1$ and $p=0.3$, resp.). A strong coupling strength will be conducive to control of the network, which is profound when the pinning ratio is large such as $p=0.3$ and above. Moreover, we take a further research on the network with coupling $c=0.1$ and $c=0.3$. From Figures 3(a) and 3(b), it can be seen that increasing the number of pinned nodes will help to synchronize the network. It is very prominent in the network with coupling strength $c=0.3$ that the network is stabilized on orbit $s(t)$ when $p=0.4$ and above. In Figure 3(a), especially a large number of pinned nodes are needed to stabilize the network with $c=0.1$ or with even smaller coupling strength. Hence, it is easier to control a strong coupling network, and only a small number of pinned nodes can synchronize the network to target orbit.

In the following, the effect of the descending CR pinning scheme on synchronization of the cortical network and the four communities is analyzed. The nodes which attain synchronization are calculated by $\sum_{i=1}^{N}\left\|x_{i}(t)-s(t)\right\|<0.01$, in which $\left\|x_{i}\right\|=\left|x_{i 1}\right|+\left|x_{i 2}\right|+\left|x_{i 3}\right|$. The results are shown in Figures $4(\mathrm{a})$ and $4(\mathrm{~b})$. Note that somatomotor community is 


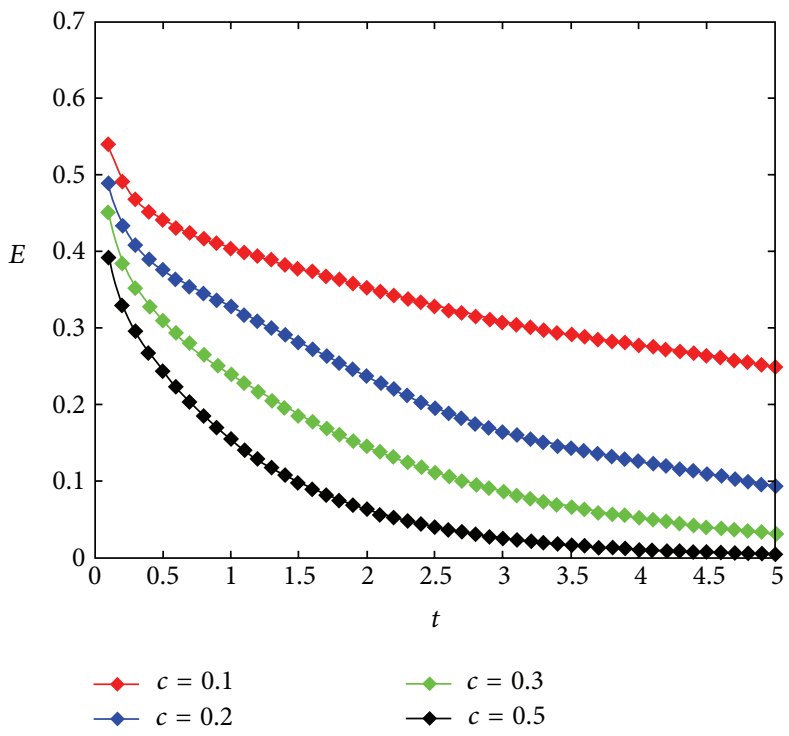

(a)

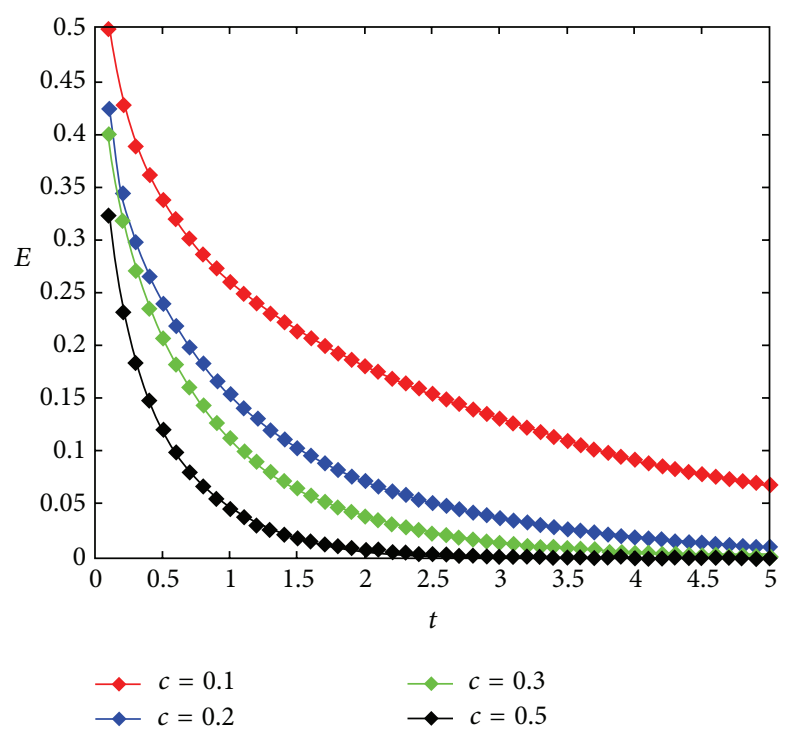

(b)

FIgURE 2: Time evolution of pinning control error $E$ for the cortical network with $N=53$, feedback gains $k=30$. With pinning ratio (a) $p=0.1$ and (b) $p=0.3$.

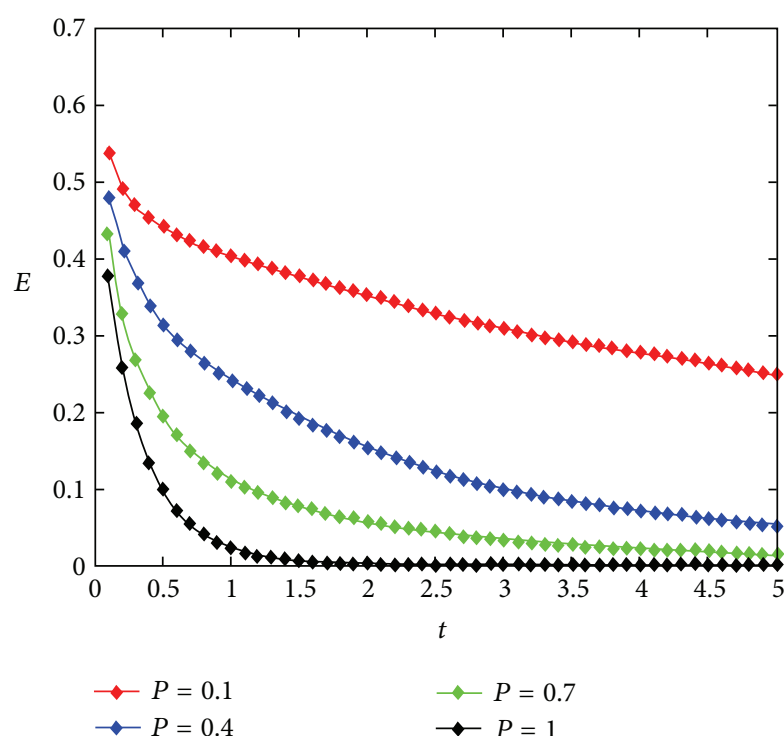

(a)

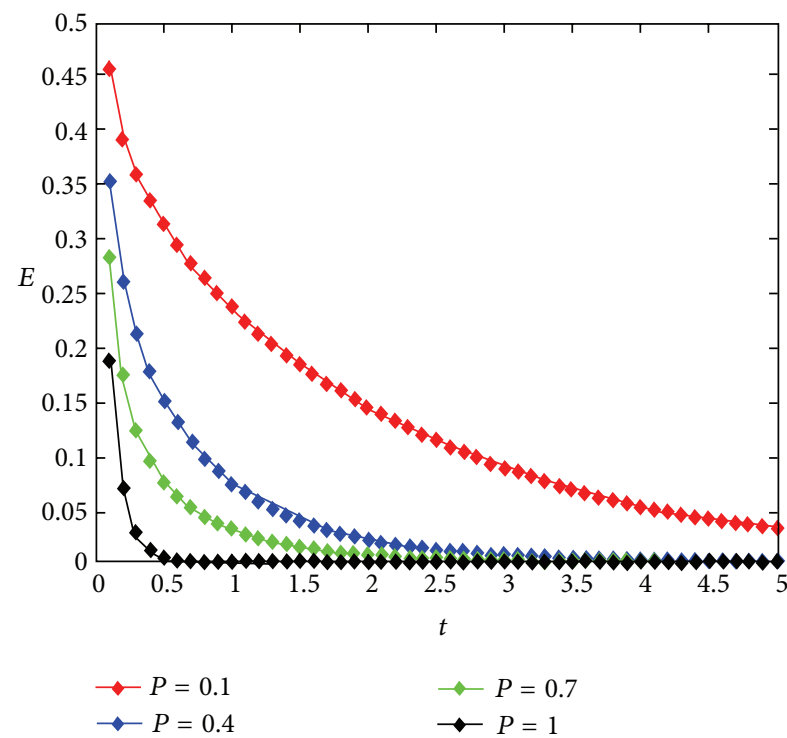

(b)

Figure 3: Control error $E$ as a function of time $t$ with different pinning ratios $p=0.1,0.4,0.7,1.0$. For the cortical network with $N=53$, the feedback gains $k=30$, and the coupling strength (a) $c=0.1$ and (b) $c=0.3$.

firstly synchronized and then visual community, frontolimbic community, and auditory community, successively. The distributions of the pinned nodes among communities are listed in Table 2. It is found that the distributions of the pinned nodes influence the dynamics of the communities. A community with a larger percentage of pinned nodes in it is easier to synchronize, for example, somatomotor community in the cortical network. Without pinned nodes inside it, the auditory is the last community to attain synchronization.

\section{Conclusions}

In this paper, the problem of pinning control of the weighted and directed cortical network has been studied. Firstly, the CR values of different areas in the cortical network are listed and then applied to analyze the hub areas in the cortical network. It is found that most of the hubs have bigger CR values than the others in the same functional community. Next, we studied the control effect of CR pinning scheme. 


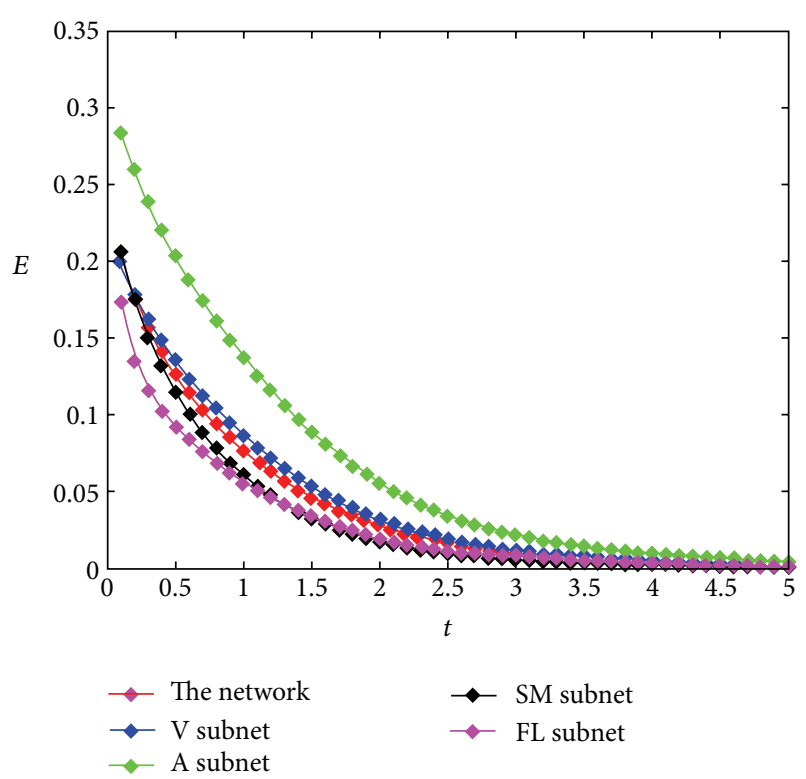

(a)

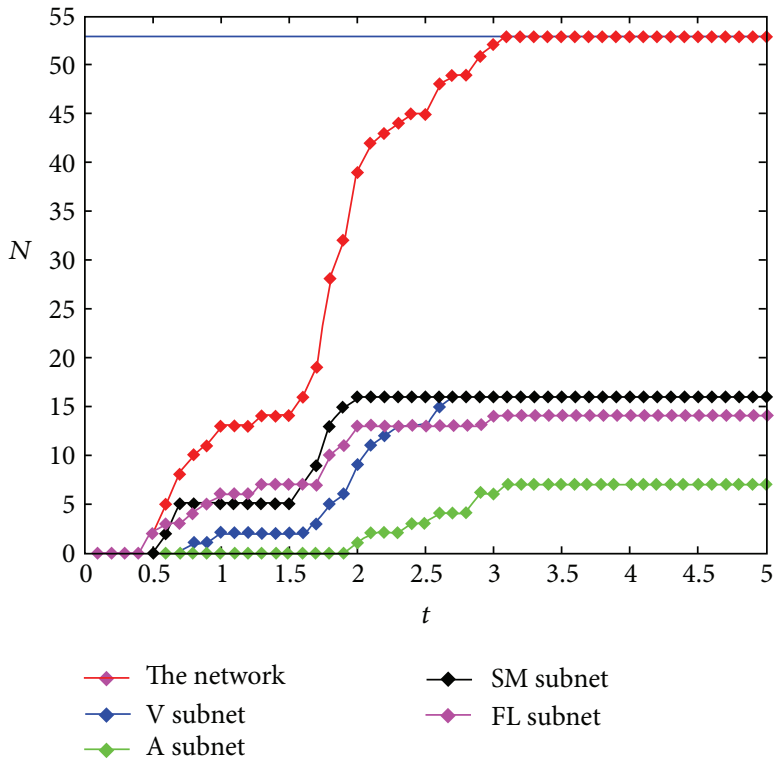

(b)

Figure 4: The CR pinning scheme of the cortical network with $N=53$, the coupling strength $c=0.3$, the pinning ratio $p=0.25$, and the feedback gains $k=30$. (a) Control error $E$ as a function of time $t$. (b) The nodes synchronized to the orbit $S(t)$ with function of time $t$. In all the plots, the legend is as follows: red (blue, green, black, and pink) lines are used in the case of the cortical network (visual community, auditory community, somatomotor community, and frontolimbic community).

TABLE 2: Pinned nodes percentage in communities with $p=0.25$.

\begin{tabular}{lcc}
\hline $\begin{array}{l}\text { Pinned nodes } \\
\text { number }\end{array}$ & $\begin{array}{c}\text { Pinned nodes percentage } \\
\text { in community }\end{array}$ & Community \\
\hline 6 & $43 \%$ & Frontolimbic \\
5 & $31 \%$ & Somato-Motor \\
2 & $13 \%$ & Visual \\
0 & 0 & Auditory \\
\hline
\end{tabular}

It is found that the coupling strength and the pinned nodes demonstrate mutual complement in controlling the network to the desired dynamics. Finally, we analyzed the global synchronization and community synchronization from the viewpoint of pinning control. We pointed out that community's synchronization is positively correlated with the number of pinned nodes inside it.

\section{Acknowledgments}

The authors acknowledge the support from German Academic Exchange Service (DAAD), the Major State Basic Research Development Program of China (973 Program, Grant no. 2010CB731400), the Key Creative Project of Shanghai Education Community (Grant no. 13ZZ050), and, the Key Foundation Project of Shanghai (Grant no. 12JC1400400).

\section{References}

[1] E. Bullmore and O. Sporns, "Complex brain networks: graph theoretical analysis of structural and functional systems," Nature Reviews Neuroscience, vol. 10, no. 3, pp. 186-198, 2009.
[2] O. Sporns, C. J. Honey, and R. Kötter, "Identification and classification of hubs in brain networks," PLOS ONE, vol. 2, no. 10, Article ID e1049, 2007.

[3] L. Zemanova, C. S. Zhou, and J. Kurths, "Structural and functional clusters of complex brain networks," Physics D, vol. 224, pp. 202-2212, 2006.

[4] C. Zhou, L. Zemanová, G. Zamora, C. C. Hilgetag, and J. Kurths, "Hierarchical organization unveiled by functional connectivity in complex brain networks," Physical Review Letters, vol. 97, no. 23, Article ID 238103, 2006.

[5] C. Zhou, L. Zemanová, G. Zamora-López, C. C. Hilgetag, and J. Kurths, "Structure-function relationship in complex brain networks expressed by hierarchical synchronization," New Journal of Physics, vol. 9, article 178, 2007.

[6] G. Zamora-López, C. Zhou, and J. Kurths, "Graph analysis of cortical networks reveals complex anatomical communication substrate," Chaos, vol. 19, no. 1, Article ID 015117, 2009.

[7] A. Arenas, A. Díaz-Guilera, J. Kurths, Y. Moreno, and C. Zhou, "Synchronization in complex networks," Physics Reports, vol. 469, no. 3, pp. 93-153, 2008.

[8] W. Zhang, Y. Tang, J. Fang, and W. Zhu, "Exponential cluster synchronization of impulsive delayed genetic oscillators with external disturbances," Chaos, vol. 21, Article ID 043137, 2011.

[9] W. Zhang, Y. Tang, J. Fang, and X. T. Wu, "Stability of delayed neural networks with time-varying impulses," Neural Networks, vol. 36, pp. 59-63, 2012.

[10] J. F. Donges, Y. Zou, N. Marwan, and J. Kurths, “The backbone of the climate network," Europhysics Letters, vol. 87, Article ID 48007, 2009.

[11] J. Lu, D. W. C. Ho, and Z. Wang, "Pinning stabilization of linearly coupled stochastic neural networks via minimum number of controllers," IEEE Transactions on Neural Networks, vol. 20, no. 10, pp. 1617-1629, 2009. 
[12] Y. Tang and W. K. Wong, "Distributed synchronization of coupled neural networks via randomly occurring control," IEEE Tratisactkms on Neural Networks and Learning Systems, 2012.

[13] P. J. Uhlhaas and W. Singer, "Neural synchrony in brain disorders: relevance for cognitive dysfunctions and pathophysiology," Neuron, vol. 52, no. 1, pp. 155-168, 2006.

[14] A. Arenas, A. Díaz-Guilera, and C. J. Pérez-Vicente, "Synchronization reveals topological scales in complex networks," Physical Review Letters, vol. 96, no. 11, Article ID 114102, pp. 1-4, 2006.

[15] X. Li, X. Wang, and G. Chen, "Pinning a complex dynamical network to its equilibrium," IEEE Transactions on Circuits and Systems, vol. 51, no. 10, pp. 2074-2087, 2004.

[16] C. W. Wu, "On the relationship between pinning control effectiveness and graph topology in complex networks of dynamical systems," Chaos, vol. 18, no. 3, Article ID 037103, 2008.

[17] L. Y. Xiang, Z. X. Liu, Z. Q. Chen, F. Chen, and Z. Z. Yuan, "Pinning control of complex dynamical networks with general topology," Physica A, vol. 379, no. 1, pp. 298-306, 2007.

[18] Q. Miao, Z. Rong, Y. Tang, and J. Fang, "Effects of degree correlation on the controllability of networks," Physica A, vol. 387, no. 24, pp. 6225-6230, 2008.

[19] W. Yu, G. Chen, and J. Lü, "On pinning synchronization of complex dynamical networks," Automatica, vol. 45, no. 2, pp. 429-435, 2009.

[20] Q. Song and J. Cao, "On pinning synchronization of directed and undirected complex dynamical networks," IEEE Transactions on Circuits and Systems, vol. 57, no. 3, pp. 672-680, 2010.

[21] F. Sorrentino, M. Di Bernardo, F. Garofalo, and G. Chen, "Controllability of complex networks via pinning," Physical Review E, vol. 75, no. 4, Article ID 046103, 2007.

[22] Y. Tang, H. Gao, W. Zou, and J. Kurths, "Identifying controlling nodes in neuronal networks in different scales," PLOS ONE, vol. 7, Article ID e41375, 2012.

[23] Y. Tang, Z. Wang, H. Gao, S. Swift, and J. Kurths, "A constrained evolutionary computation method for detecting controlling regions of cortical networks," IEEE/ACM Tratisactkms on Computational Biology and Bioinformatics, vol. 9, pp. 1569-1581, 2012.

[24] Y. Tang, H. Gao, J. Kurths, and J. Fang, "Evolutionary pinning control and its application in UAV coordination," IEEE Transactions on Industrial Informatics, vol. 8, pp. 828-838, 2012.

[25] Y. Y. Liu, J. J. Slotine, and A. L. Barabási, "Controllability of complex networks," Nature, vol. 473, no. 7346, pp. 167-173, 2011.

[26] M. Egerstedt, "Complex networks: degrees of control," Nature, vol. 473, no. 7346, pp. 158-159, 2011.

[27] Y. Y. Lu and X. F. Wang, "Pinning control of directed dynamical networks based on Control Rank," International Journal of Computer Mathematics, vol. 85, no. 8, pp. 1279-1286, 2008.

[28] C. C. Hilgetag, G. A. P. C. Burns, M. A. O’Neill, J. W. Scannell, and M. P. Young, "Anatomical connectivity defines the organization of clusters of cortical areas in the macaque monkey and the cat," Philosophical Transactions of the Royal Society B, vol. 355, no. 1393, pp. 91-110, 2000.

[29] G. Zamora-Lopez, C. S. Zhou, and J. Kurths, "Cortical hubs form a module for multisensory integration on top of the hierarchy of cortical networkse," Frontiers in Neuroinformatics, vol. 4, pp. 1-13, 2010. 


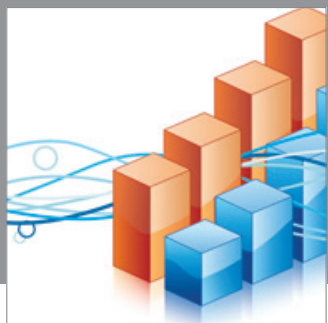

Advances in

Operations Research

mansans

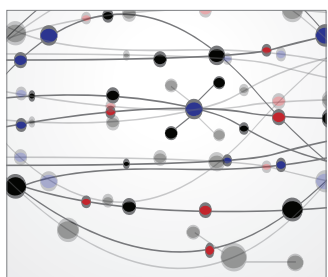

The Scientific World Journal
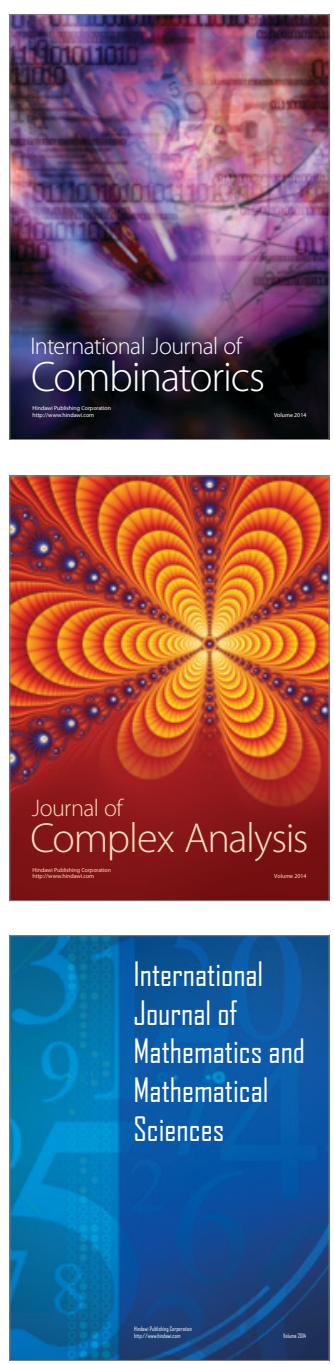
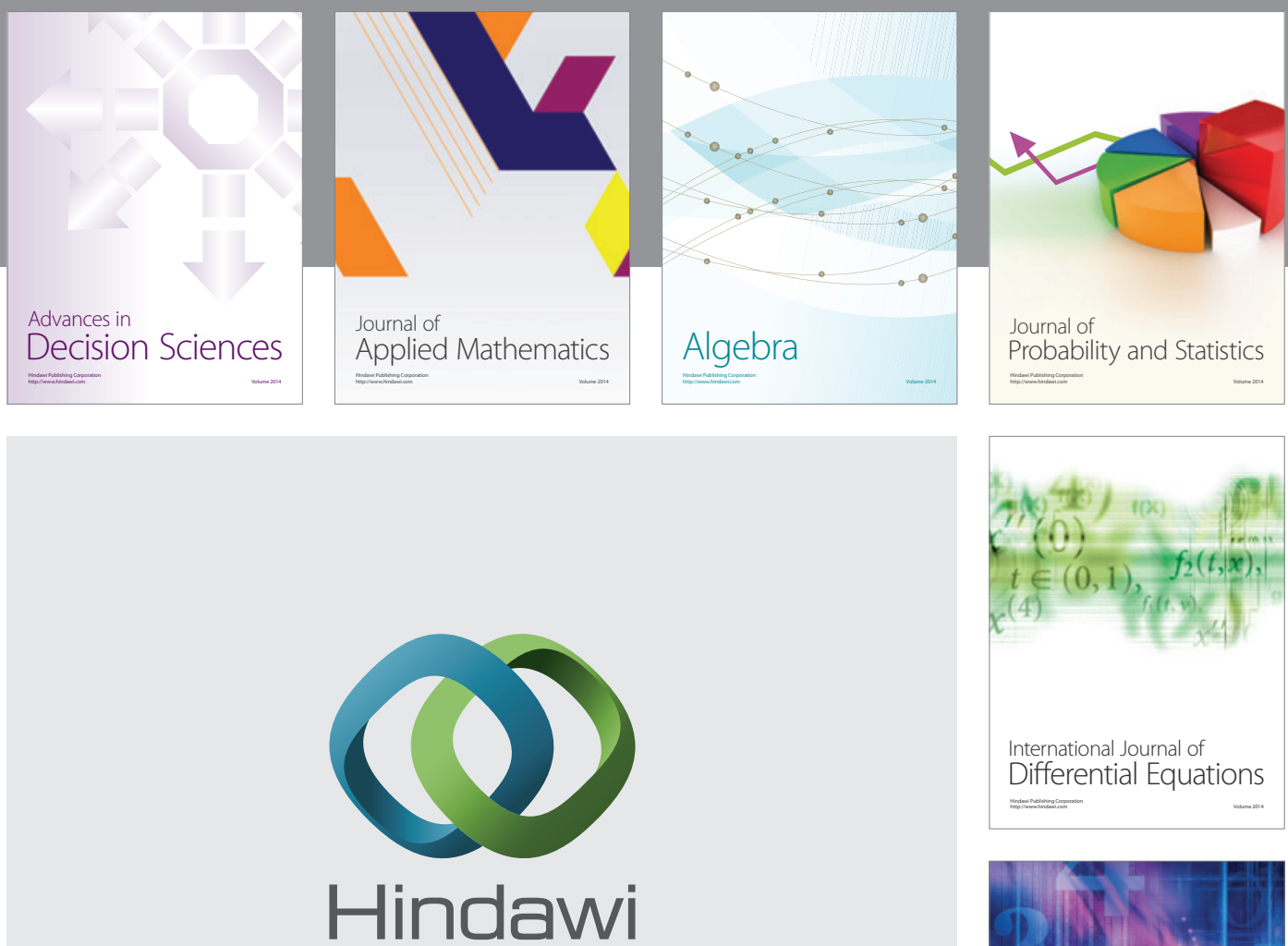

Submit your manuscripts at http://www.hindawi.com
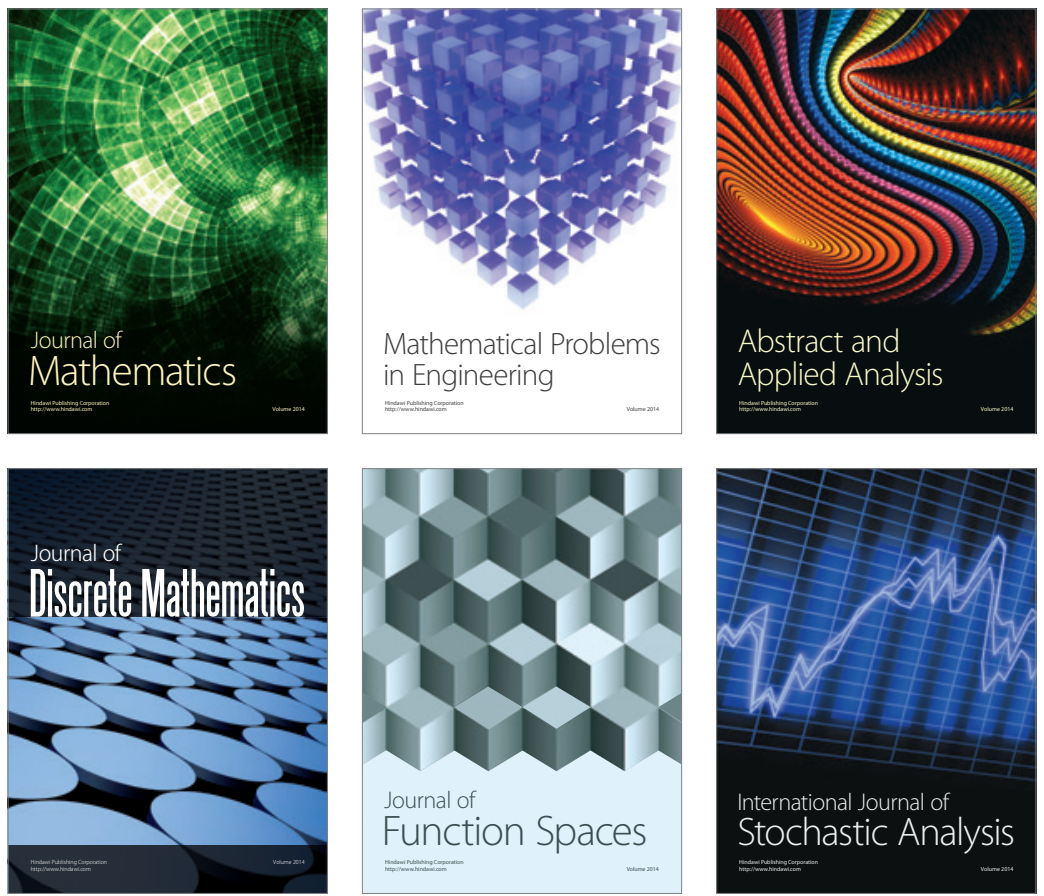

Journal of

Function Spaces

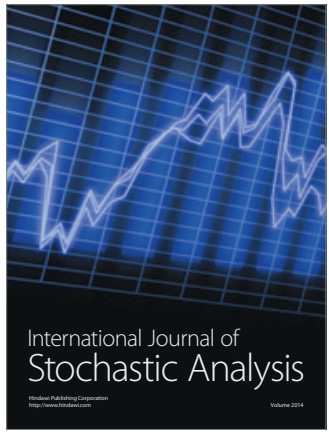

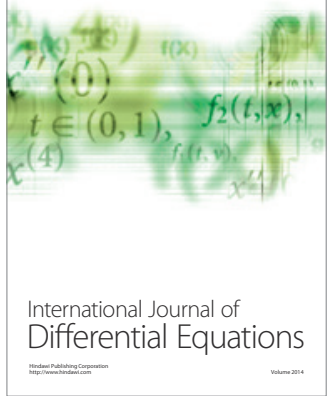
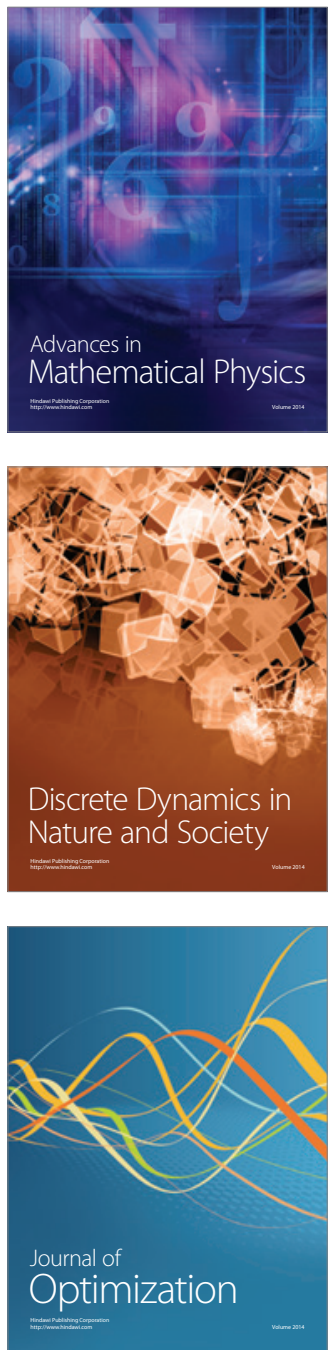